\title{
Priming effects with ambiguous figures
}

\author{
Paula Goolkasian and Courtney Woodberry \\ University of North Carolina, Charlotte, North Carolina
}

\begin{abstract}
We varied the format and semantic content of primes to determine the degree to which they would influence the interpretation of ambiguous figures. The primes were objects or object names that were related in some way to one of the two organizations of the ambiguous figures. In Experiment 1, we provided some normative data regarding the stimulus materials, whereas in Experiment 2, an orienting question was used to focus attention on the semantic relationship between the prime and the figure. In Experiment 3, we used the orienting question to divert attention away from the relationship by asking about physical features of the figures. Recognition responses to biased versions of the figures and to new figures were measured. Primes that were loosely and indirectly associated with one of the two interpretations of an ambiguous figure were found to be effective at biasing the interpretation of an ambiguous figure in the direction of the primed alternative but only if attention was focused on the semantic relationship between the two stimuli. Attention to the physical characteristics of the stimuli during encoding eliminated the prime's influence on complex object perception. These findings are consistent with the conceptual priming literature and extend those of some recent studies (Balcetis \& Dale, 2007; Feist \& Gentner, 2007), which show that the interpretation of complex figures can be biased by the advanced presentation of related verbal information.
\end{abstract}

Pictures that can be interpreted in more than one way have intrigued researchers in visual perception since the early days of psychology. Long and Toppino (2004), in a review, suggested that a variety of sensory and cognitive processes are involved in explaining exactly how the same image can be organized in more than one way. These processes work in both top-down and bottom-up ways to influence what we see. In the present experiments, we focus on factors that are associated with cognitive set.

Balcetis and Dale (2007) investigated set effects by having participants read several paragraphs of material that were loosely and indirectly associated with one of the two interpretations of an ambiguous figure. When the participants were shown the ambiguous figure and asked to describe it, their responses were biased in the direction of the verbal material that appeared as the prime. This finding is important because it demonstrates the effects of cognitive set with a verbal rather than a visual prime, and in addition, the prime that is found to bias the interpretation of the figure is indirect and loosely associated with one of the versions of the figure. Previous work had not shown consistent priming with verbal material. For example, Leeper (1935) compared verbal and visual set by priming with a paragraph that described one of the organizations of the ambiguous figure with the biased figure itself. Verbal priming worked for one ambiguous figure but not for a second example that was tested, whereas visual priming was effective for both figures. Balcetis and Dale's findings differed from Leeper's in that they showed consistent priming effects across several ambiguous figures, and they used paragraphs that provided a verbal context for one of the two organizations of the figure but that did not describe the figure directly.

Although set effects have been demonstrated before, they resulted primarily from direct perceptual effects. For example, it has long been known that presenting a biased version of a figure in advance of the ambiguous one is an effective way of influencing the participants' interpretation of the picture (Botwinick, 1961; Goolkasian, 1987; Leeper, 1935). Also, Bugelski and Alampay (1961) showed that presenting a picture that is related to the biased version of the figure is sufficient to influence the interpretation of the ambiguous figure. As long as the prime is from the same perceptual set as the figure, it can influence the interpretation of the ambiguous figure.

There is a distinction in the research literature (Farah, 1989; Matsukawa, Snodgrass, \& Doniger, 2005; Tulving $\&$ Schacter, 1990) between perceptual priming, which is direct and based on the physical properties of a stimulus, and conceptual priming, which is indirect and based on semantic memory. Tulving and Schacter showed evidence that direct priming operates at an early, presemantic level of processing that is associated with a perceptual representation system, whereas conceptual priming is based on memory and is associated with a late processing stage. Although both types of priming facilitate the recognition of a targeted stimulus, Farah argued that they do so by means of qualitatively different effects on attention. Perceptual priming affects sensitivity changes, whereas conceptual priming creates a bias to respond before the target is fully

P. Goolkasian, pagoolka@uncc.edu 
encoded. Matsukawa et al. compared the effectiveness of these types of priming on a picture identification task and found an advantage for conceptual primes. Names or images of the target that are conceptual, or a combination of perceptual and conceptual, facilitated target identification, whereas purely perceptual primes that were similar in shape to the target did not.

Balcetis and Dale's (2007) finding of a verbal priming effect is consistent with the research in conceptual priming and with some evidence from research in linguistics showing that language can influence visual perception. Feist and Gentner (2007) presented ambiguous pictures with and without sentences that biased the spatial relationship between objects in visual scenes. They found significant differences between the two groups in their recognition response. The group with the sentences produced more false alarms than the group without to the version of the picture that demonstrated the spatial relationship primed by the sentences. Their findings were interpreted to show that language interacts with visual information to influence the way a picture is encoded and remembered.

An interactive explanation in which top-down knowledge guides low-level processes has also been endorsed by researchers who have found influences of previous experience on the perception of ambiguous figures. Most notably, in the work of Peterson and colleagues (Peterson \& Gibson, 1994; Peterson, Harvey, \& Weidenbacher, 1991), shape recognition processes were found to influence both initial figure-ground responses and the reversal of figure-ground.

In the present work, we also looked at the effect of language on visual perception, but we did so within the context of cognitive set. We believe that the success of the priming lies in the semantic relationship between the prime and the visual object and not necessarily in the format of the prime. Whether the prime is verbal, visual, direct, or indirect is not as important as its perceived relationship to the visual object. When presented, the prime must be linked to one of the two organizations of the figure, so that its appearance directs the participants toward those elements in the figure that are associated with only one of the organizations.

Consistent with the work of Balcetis and Dale (2007), we tested the effectiveness of primes that are related in some way to one of the two organizations of ambiguous figures. However, rather than using just verbal primes, we compared verbal and visual primes that were equated for content. The primes were objects (or objects' names) that were related by either association or category membership to one of the two organizations of the figures. For example, to prime the squirrel version of the swan/squirrel figure, we used acorn and squirrel feeder. Primes varied in number from one to three to provide a sufficient context for one of the two interpretations of the figure. Experiment 1 was conducted to measure the degree of relatedness between the primes and the two interpretations of the ambiguous figures and to test whether the picture and word primes were related in similar ways to the figures.

Figure 1 provides some examples of the ambiguous figures that were used as stimuli. For the most part, they represent two of the three types of ambiguous figures described by Peterson (1993). For some, like the swan/ squirrel and the Eskimo/Indian, the reversal involves a reconstrual of the parts of the figure. The squirrel's tail becomes the swan's head, and the Indian's face reverses into the Eskimo boy's back. Other figures involve a redetermination of the figure-ground relationship. In the arrow figure, left-pointing yellow arrows appear against a black background, or right-pointing black arrows appear against a yellow background. Similarly, in the liar/face figure, the word liar appears in white letters with a slanted orientation, or the profile of a man's face with eyeglasses appears against a black background. In all cases, the reversals change the meaning of the figure. We did not select figures like the Necker cube (Necker, 1832) that involved fluctuations in depth, and when possible, we tried to stay away from classic figures like Boring's wife/mother-in-law figure (Boring, 1930) or the face/vase (Rubin, 1958) that are so familiar to students. Because knowledge of an alternative interpretation of the figures has been shown by Rock and his colleagues (Girgus, Rock, \& Egaz, 1977; Rock \& Mitchener, 1992) to be an important aspect to seeing the reversal, we tried to use figures that were not widely distributed. In these studies, it was found that if observers are not familiar with the ambiguous figures and not told about them in the instructions, they may not experience the reversal. By minimizing past associations that the participants may have had with the pictures, we studied in a relatively unbiased manner whether the prime would affect the interpretation of the ambiguous figure. For most of the figures, the two interpretations are not equally likely. To make sure that our effects were genuine and representative of how complex figures are processed, we averaged the participants' responses across several figures and primed both interpretations of the ambiguous figures.

\section{EXPERIMENT 1}

Experiment 1 was run to gather some normative data about the stimulus materials that we wanted to use in the two following experiments. To find a sufficient number of ambiguous figures, we selected examples from past research studies, as well as from those available over the Internet. When they were available from the original source, we also copied the two alternative (biased) versions of the ambiguous figure. However, there were several that we needed to produce by either enhancing or minimizing the features of the ambiguous figure. In Experiment 1, we measured the participants' interpretations of the biased versions of the ambiguous figures and assessed the degree of relatedness between the primes and the biased versions of the figures. We were also interested in measuring the similarity in the participants' ratings to picture and word versions of the primes.

\section{Method}

Participants. The participants were 38 (71\% female) students from the University of North Carolina, Charlotte, who were at least 18 years of age and had no history of visual impairments. They participated in the experiments to obtain extra credit points toward their psychology class grade. Data from 3 of the participants were incom- 


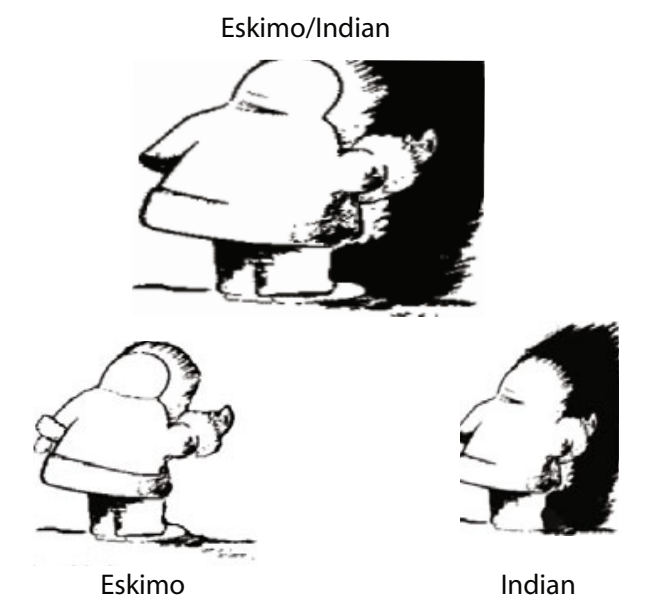

Primes

Young Boy

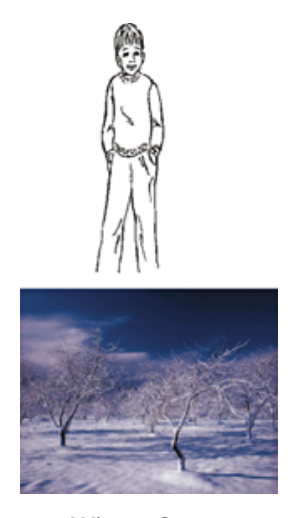

Winter Scene

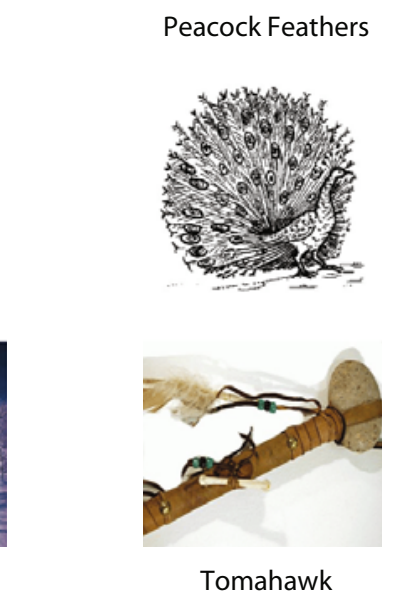

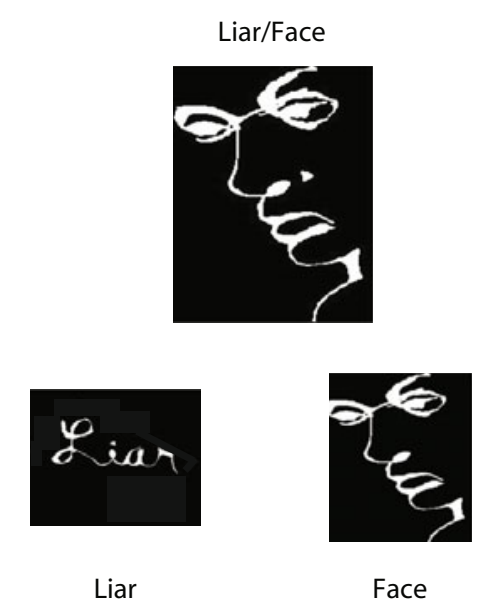

Primes

Figure 1. Samples of the stimulus items. The Eskimo/Indian and liar/face ambiguous figures are presented together with the biased versions of the figures and their respective picture and word primes.

plete because of a difficulty with numbering the responses and, as a result, were not included in all of the analyses that are reported in the Results section.

Materials. A list of the 18 ambiguous figures used to develop the stimulus material is provided in the Appendix, and the figures themselves are available from the author. These figures were selected from past research studies, as well as from those available over the Internet via a Google search. For each of the figures, there were two alternative interpretations that involved a change of meaning and either a reconfiguration of the parts of the figure or a reversal in figure-ground organization. In the biased versions, the ambiguous figure was altered in such a way that one of the two interpretations was the more prevalent. Figure 1 provides some examples. When available from the original source, we copied the two biased versions of the ambiguous figure. In all other cases, we produced them with Adobe Photoshop by copying the ambiguous figure, enhancing the features or visual detail of one of the two interpretations, and minimizing the features of the other interpretation by erasing some of the visual detail. Although some of the student participants may have encountered the figures previously, we tried to choose examples that were novel or not as familiar as some of the classic figures, such as the wife/mother-in-law (Boring, 1930) or the face/vase (Rubin, 1958) figures that are used in discussions of ambiguous figures for general psychology courses. The stimuli were pasted into Adobe Photoshop to adjust the figure sizes to a standard, which varied from a minimum of $5 \times 5 \mathrm{~cm}$ to a maximum of $9 \times 9 \mathrm{~cm}$, and saved as GIF files. The participants sat 18 in. from the monitor.

We also developed a set of primes that were either related or unrelated to the two interpretations of the ambiguous figures. The related primes were one, two, or three objects or object names that were associated in varied ways with one of the two organizations. Some of the prime-figure pairings were related by association, whereas others represented categorical relationships. The number of objects used for a given prime depended on our judgment of what was necessary to provide a sufficient context. Two primes were used for most of the figures, but in two examples three primes were needed, and in four instances only one object was used. In some instances, a single object (such as words on a page) included multiple elements that were associated with the target figure. The unrelated primes were the same objects but paired with an ambiguous figure whose two organizations were not related in any semantic way to the prime. A list of the related primes used for each of the figures is provided in the Appendix. Two versions of the primes matched in content were produced: a picture and a word version. The primes that appeared as words were uppercase characters in 24-cpi Geneva font. The pictures were imported into Adobe Photoshop, and their sizes were adjusted to approximately $4 \times 4 \mathrm{~cm}$.

The stimuli were displayed on a 15 -in. flat-screen monitor. Stimulus presentation and data collection were controlled by SuperLab version 4.0 running on a Macintosh G4 computer. Two versions of 
the stimulus files were developed: one with the primes presented as pictures and the other with the primes presented as words.

Procedure. The participants were randomly assigned to view the primes in either a picture or word format and were run individually in 15-min sessions that consisted of two phases. Instructions indicated that the experiment dealt with picture perception.

In the first phase, the participants were shown three practice pictures, followed by a random list of the 36 biased versions of the ambiguous figures, and were asked to describe each picture with a word or two. The pictures appeared one at a time, and the participants were allowed to view them for as long as necessary before writing their answer on a response sheet. Each response sheet had 39 numbered lines: one for each of the stimulus items.

In the second phase of the study, the prime appeared for $5 \mathrm{sec}$, followed by a biased version of the figure, and the participants used a 6 -point numerical rating scale $(1$, not related; 6 , related $)$ to indicate the degree of relatedness between the prime and the biased figure. The participants responded by using the first six keys on the numerical keypad. There were 72 trials, in which each of the 36 biased versions of the ambiguous figures appeared twice, once with a related and once with an unrelated prime. The presentation order of the 72 trials was randomly determined for each of the participants.

\section{Results}

Written responses to each of the biased figures were categorized by two independent raters as consistent with the biased figure, consistent with the alternate version of the ambiguous figure, containing elements of both versions, and other. The results from the two raters were averaged, and the mean proportion of times across participants that the responses fell into each of the categories are, respectively, $.70, .08, .08$, and .14 . The categorization responses from the two raters of the proportion of times across participants that the biased figures were identified appropriately were correlated $[r(34)=.91, p=.01]$.

Twenty-one of the figures were identified by over $60 \%$ of the participants with words that were consistent with the way that we labeled the figure. The 11 figures that were identified somewhat less consistently were redrawn before they were used as stimuli in Experiments 2 and 3. The redrawn figures were pilot tested a second time and were included in the follow-up studies only when $60 \%$ of the respondents identified them with words that were consistent with the biased figure. All 32 biased figures were used in the follow-up studies.

The ratings of the degree of relatedness between the prime and the biased versions were analyzed using participants $\left(F_{1}\right)$ and items $\left(F_{2}\right)$ as random factors. In the analysis over participants, format was a between-groups factor, and the within-groups factor of prime condition included three conditions: related to the first version, related to the second version of the ambiguous figure, and unrelated to either. Because the data from 3 participants were removed because of incomplete responses, there were 19 participants who received the picture primes and 16 who received the word primes. In the analysis over items, first or second alternative version was a between-groups factor, and prime type (related or unrelated) and prime format (picture or word) were the within-groups factors.

In both of the analyses, there were significant main effects of prime type $\left[F_{1}(2,66)=241.00, p<.01 ; F_{2}(1,34)=\right.$ $262.47, p<.01]$ and prime format $\left[F_{1}(1,33)=10.70\right.$,
Table 1

Ratings of the Relationship Between the Prime and the Biased Figures Across Conditions

\begin{tabular}{lll}
\hline \multicolumn{1}{c}{ Condition } & $M$ & $S D$ \\
\hline Related picture prime & 4.7 & 0.82 \\
Related word prime & 4.1 & 0.98 \\
Unrelated picture prime & 2.1 & 0.69 \\
Unrelated word prime & 1.6 & 0.35 \\
\hline
\end{tabular}

Note-Ratings were made on a 6-point scale (1, unrelated; 6 , related $)$.

$\left.p<.01 ; F_{2}(1,34)=33.68, p<.01\right]$. Table 1 presents the mean ratings for each of these effects. As was expected, related primes were rated more highly than unrelated primes, and there was a format difference. The picture primes were rated more highly than were the words, with a difference of around .5 of a scale point. The prime type did not interact with format, however $\left[F_{1}(2,66)=1.13\right.$, $\left.p=.33 ; F_{2}(1,34)=1.15, p=.29\right]$. Ratings were not found to differ between the two versions of the figures, and this variable did not interact with prime type or prime format.

From the analysis over items, individual item ratings were printed and used to determine whether or not changes were needed to the prime-figure pairings before use in Experiments 2 and 3. Changes were made to related primes that were rated on average to be lower than 4 and to unrelated primes that were rated to be higher than 3 . For the related primes, the changes involved finding objects that provided a better context for the figure. In the lady/ man example, the prime for the lady was changed to a picture of a lady seated on the floor, because the previous picture of a lady was judged to be unrelated. Changes to verbal descriptions that were used as primes were also made for a few items when the word primes were rated much lower than the picture primes. Ratings to the picture and word versions of the primes were correlated whether related $[r(34)=.56, p<.01]$ or unrelated $[r(34)=.51$, $p<.01]$. Also, ratings of the strength of the relationship among the related pairs were not found to be associated with the number of objects used as primes $[r(34)=.19$, $p=.30]$.

\section{Discussion}

In general, the results were successful in providing some normative data for the stimulus material used in the next two experiments. Twenty-one of the 36 biased figures were labeled in a manner consistent with our expectations by at least $60 \%$ of the participants. The 11 figures that did not meet this criterion were redrawn until they met the criterion.

Similarly, the findings also validated our assumptions about the relatedness between the primes and the alternative versions of the figures. Changes were made to any prime-figure pairing when average ratings were less than 4 in the related condition and greater than 3 in the unrelated condition. For the final set of stimuli, mean ratings for the 36 related pairings ranged from 4.05 to 5.68, indicating variability in the strength of the pairings, with some weak, medium, and strong relationships. 


\section{EXPERIMENT 2}

In our priming study, we also wanted to investigate how important it was for the participants' attention to be focused on the relationship between the prime and the visual object. Mulligan and Peterson (2008) examined the relationship between attention and implicit memory and found that priming was affected by selective attention. Also, Ramponi, Richardson-Klavehn, and Gardiner (2007) found that conceptual priming was affected by depth of processing. In particular, weak and medium associates showed depthof-processing effects. In Experiments 2 and 3, an orienting question was used during the study trials to vary the degree to which the participants paid attention to the semantic relationship between the prime and the ambiguous figure. Questions that focus on physical features of the stimuli promote shallow processing, whereas those that focus on semantic relationships promote deep processing (Craik \& Lockhart, 1972; Craik \& Tulving, 1975; Intraub \& Nicklos, 1985). We manipulated levels of processing between Experiments 2 and 3 to direct the allocation of attention while the participants encoded the ambiguous figures. If, as some have suggested (Tulving \& Schacter, 1990), conceptual priming is based on memory and operates at a later rather than an earlier stage of processing, highlighting the semantic relationship between the prime and one of the two organizations of the ambiguous figure would be expected to influence how that figure is encoded. With a focus on the surface features of the prime and the ambiguous figures, however, we did not expect to be able to influence the encoding of the ambiguous figure.

Our work differed from other recent priming studies (Balcetis \& Dale, 2007; Feist \& Gentner, 2007) in that we varied the format, the semantic content of the prime, and the attention paid to the semantic relationship between the prime and the ambiguous figure. The semantic content of the prime was varied by manipulating whether the prime was related or unrelated to one of the two organizations of the figure. As they were in Balcetis and Dale's study, the relationships were loose and not consistently defined. Any association to one of the organizations of the figures, whether direct or indirect, was sufficient. The findings from Experiment 1 show that across figures, there is some variation in the strength of the prime-figure pairings. Effective priming would be evident if the presence of the related prime was found to influence the participants' organization of the ambiguous figure in comparison with the unrelated prime.

We varied the format to test whether the priming effect obtained by Balcetis and Dale (2007) and Feist and Gentner (2007) was specific to the presentation of verbal material or resulted from any related information presented either as a picture or as words. The results from Matsukawa et al. (2005) suggest that it is the semantic relatedness of the prime rather than the prime format that determines its effectiveness. Also, some research shows that perceptual symbols are activated in language comprehension. Zwaan, Stanfield, and Yaxley (2002) found faster naming and recognition responses to line drawings when the depicted ob- jects were mentioned in a preceding sentence. Priming effects that are format specific would support an effect that resulted from the perceptual processing of either linguistic or visual information. Priming effects that occur with both formats, however, would suggest a conceptual basis for the effect-one that resulted from a top-down process. Such a finding would extend Feist and Gentner's interactive encoding hypothesis to include both verbal and visual primes and would be consistent with other work showing that prior knowledge and/or experience influences the perception of ambiguous figures (Peterson \& Gibson, 1994; Peterson et al., 1991).

In Experiment 2, we focused attention on the semantic relationship between the prime and the figure by requiring participants to respond to an orienting question about the relatedness of the information during encoding. The prime appeared for $5 \mathrm{sec}$, followed by the ambiguous figure. The participants' yes-no response terminated the presentation of the ambiguous picture. In Experiment 3, we deemphasized the relatedness of the two screens by changing the orienting question to one about surface features. We probed whether the screens were black and white, colored, or mixed. Priming effects were expected in Experiment 2 but not in Experiment 3. By diverting attention away from the prime's association to one of the organizations of the ambiguous figures, we expected to attenuate or remove the influence of the prime.

A recognition memory test was used in the test phase of the experiment. The participants were shown the biased versions of the ambiguous figures, together with some new figures, and asked whether they had appeared during the study phase. The new figures included new examples of the primed shape (for the swan/squirrel figure, a new picture of a swan), as well as some new items that were unrelated to the set of ambiguous figures. Following a technique introduced by Bar and Biederman (1998), the new shapes were included to measure false alarm rates to the primed shape. We wanted to control for the possibility that the participants were responding to the test items on the basis of their memory of the primed shape rather than their memory of the primed version of the ambiguous figure. Effective priming would be demonstrated by higher recognition of the biased version of the figure that was primed with related rather than unrelated information. The unprimed versions of the figures were also included in the recognition test for comparison with the other conditions. Because the recognition response represented a nonverbal way of assessing how the participants encoded the ambiguous figures during the study trials, it provided some control for the linguistic bias that is inherent in studies in which participants are asked to describe what they see.

\section{Method}

Participants. Sixty students ( $77 \%$ female) from the University of North Carolina, Charlotte, who were at least 18 years of age and had no history of visual impairments, volunteered to obtain extra credit points toward their psychology class grade. None of these students had participated in Experiment 1.

Materials. A list of the 18 ambiguous figures that were used as the stimulus material during the study phase is provided in the Ap- 
pendix, and the figures are available from the author. The stimuli were pasted into Adobe Photoshop to adjust the figure sizes to a standard, which varied from a minimum of $5 \times 5 \mathrm{~cm}$ to a maximum of $9 \times 9 \mathrm{~cm}$, and saved as GIF files. The participants sat $18 \mathrm{in}$. from the monitor.

For the study trials, we used the set of primes that were tested in Experiment 1. Related primes were rated on average with scores ranging from 4 to 6 on a 6-point relatedness scale, whereas the unrelated primes were rated on average with scores between 1 and 3 . A list of the primes used for each of the figures is also provided in the Appendix.

The 36 biased versions of the ambiguous figures tested in Experiment 1 were used as the stimuli during the test phase. In the biased versions, the ambiguous figure was altered in such a way that one of the two interpretations was more prevalent. Biased versions were identified with words consistent with our labeling by more than $60 \%$ of the participants. Also developed were 36 new shapes that represented new examples of the primed shapes and 12 new items that were similar in size, content, and visual appearance to the biased versions of the ambiguous figures.

The stimuli were displayed on a 15 -in. flat-screen monitor. Stimulus presentation and data collection were controlled by SuperLab version 4.0 running on a Macintosh G4 computer.

Procedure. The participants were run individually in 30-min sessions that consisted of a study and a test phase. Instructions indicated that the experiment dealt with the effects of primes on picture perception. The participants were not informed about the use of ambiguous figures until the debriefing session at the end. There was a posttest question about whether the participants were aware that ambiguous figures had been used during the study trials.

Study phase. The participants were randomly assigned to view the primes in either a picture or a word format. On each trial, an orienting question, about whether the information on the next two screens was related, appeared for $1 \mathrm{sec}$. It was followed by the prime(s) for $5 \mathrm{sec}$ and then the ambiguous figure. The figure remained on the screen until the participants made a yes-no response (with either the " $\mathrm{F}$ " or the "J" key on the keyboard). A note under the monitor made the yes-no key labels visible at all times to the participants. There were 18 trials in the study phase, evenly divided across the three priming conditions. Each participant saw the 18 ambiguous figures once, and for six of the trials, the figure was preceded by a prime (or primes) that was related to the first interpretation of the ambiguous figure; for six of the trials, the prime was related to the second interpretation; and for the remaining trials, the prime was unrelated to either of the interpretations. Designation of first or second interpretation was arbitrarily assigned to each figure. Three versions of the stimulus lists were created for each of the two format conditions so that we could counterbalance the presentation of each of the priming conditions across each of the ambiguous figures. Across participants, all 18 figures were presented once in each of the priming conditions. In total, six versions of the stimulus lists were created to reflect the between-groups manipulation of presentation format and the within-groups effect of prime condition. Ten participants were randomly assigned to each of the six stimulus lists.

Test phase. Following the study phase, a distractor task was performed for $2 \mathrm{~min}$. The participants were asked to start at 100 and count backward out loud in units of 3. A surprise recognition memory test followed. On each trial, the participants were shown the biased versions of the ambiguous figures, new shapes, or new items one at a time and asked to indicate whether it had been presented during the study trials. The participants responded using a 6-point scale $(1$, very confident no; 2 , somewhat confident no; 3 , not very confident no; 4 , not very confident yes; 5 , somewhat confident yes; 6 , very confident yes). There were 60 recognition trials composed of the two biased versions of the ambiguous figures used during the study trials, 12 new shapes that represented new versions of the primed shape, and 12 new items. The trials were presented in a different random order for each of the participants.

\section{Results}

Mean recognition scores for each of the experimental conditions were calculated across participants, and these data are reported in Table $2 .{ }^{1}$ A $2 \times 5$ ANOVA was used to test for the between-groups effect of prime format and the within-groups effect of test condition. Mean recognition scores were also calculated across items, and a second analysis tested for the format and test condition effects with these data. However, the test condition effect only included three of the five item types. Responses to the new figures (new shapes and new items) were excluded, because the individual items varied across the six stimulus lists. The $F$ tests that are reported for all of the withingroups effects include the Greenhouse-Geisser correction when necessary to protect against possible violation of the sphericity assumption.

Both analyses showed significant effects of test condition $\left[F_{1}(4,232)=290.73, p<.01, \eta^{2}=.83 ; F_{2}(2,70)=\right.$ $\left.6.29, p<.01, \eta^{2}=.15\right]$. Follow-up within-subjects contrasts (at the $p<.05$ significance level) show that with both sets of data, recognition of the biased versions of the ambiguous figures was significantly greater when primed with a related rather than an unrelated prime or when the alternative versions were primed. The latter two conditions did not differ from each other. The largest differences, however, were between the new figure conditions (new shape and new item) and the biased versions of the ambiguous figures (labeled as primed, alternative primed, and unrelated primed in Table 2). New shapes were also falsely recognized more readily than the new items that were unrelated to the ambiguous figures. The participants were clearly able to discriminate the new figures, irrespective of whether they were new examples of the primed shape or not, from the biased versions of the ambiguous figures that were shown during the study trials.

Format of the prime did not affect recognition $\left(F_{1}<1\right)$; however, in the analysis over items, the effect was marginal $\left[F_{2}(1,35)=3.77, p=.06\right]$, with higher recognition scores associated with the picture format condition. However, there was no format $\times$ prime type interaction in either of the analyses $\left[F_{1}(4,232)=1.18, p=.32 ; F_{2}<1\right]$.

$\boldsymbol{A}^{\prime}$ Analysis. To control for the participants' response bias, we calculated $A^{\prime}$ scores (nonparametric measures of sensitivity developed by Grier, 1971) from the recognition data. In our calculations, we used the data collected in the

Table 2

Recognition Responses for Each of the

Experimental Conditions in Experiment 2

\begin{tabular}{|c|c|c|c|c|}
\hline \multirow[b]{2}{*}{ Condition } & \multicolumn{2}{|c|}{ Picture Prime } & \multicolumn{2}{|c|}{ Word Prime } \\
\hline & $M$ & $S D$ & $M$ & $S D$ \\
\hline Primed & 4.02 & 0.89 & 3.87 & 0.80 \\
\hline Alternative primed & 3.53 & 0.98 & 3.54 & 0.93 \\
\hline Unrelated primed & 3.67 & 0.93 & 3.49 & 0.74 \\
\hline New shape & 1.78 & 0.66 & 1.40 & 0.66 \\
\hline New item & 1.36 & 0.44 & 1.34 & 0.52 \\
\hline
\end{tabular}

Note-Recognition responses were measured on a 6-point response scale (1, very confident no; 2 , somewhat confident no; 3 , not very confident no; 4 , not very confident yes; 5 , somewhat confident yes; 6 , very confident yes). 
analysis across items to deal with a concern raised by Hollingworth and Henderson (1998) regarding the importance of comparing the detection of a particular signal when it was present and the false detection of the same signal when it was not present. In our application, we computed the hit rate by using the proportion of times that the participants produced a yes response (rating scale response greater than 3) to each of the biased versions of the ambiguous figures when it was primed with related information and compared it with the proportion of times that the participants produced a yes response to the same biased version when it was primed with unrelated information (False Alarm 1) and with the proportion of times that the participants produced a $y$ es response to the same biased version when it was the unprimed alternative version of the ambiguous figure (False Alarm 2). The $A^{\prime}$ scores were averaged across formats and all 36 of the biased items and were found to be significantly greater than chance $(.5)$. The analysis indicated that priming worked to influence recognition rate whether compared with False Alarm 1, when the same figure was primed with an unrelated prime $\left[A_{1}^{\prime}=.55, t(35)=2.37, p=.01\right]$, or with False Alarm 2, when the alternative interpretation was primed $\left[A_{2}^{\prime}=.57, t(35)=3.30, p<.01\right]$.

After the experimental session, the participants were asked whether they were aware that ambiguous figures were used during the study phase. Only $12 \%$ of the participants indicated that they were unaware. When the data for the unaware participants were analyzed separately, the same pattern of findings was obtained; that is, figures primed with a related stimulus were recognized at a higher rate than figures primed with an unrelated prime or when the alternative version was primed.

Study trial responses. As a manipulation check, we also looked at the proportion of correct responses to the study trials to determine whether the participants correctly identified the related and unrelated prime-figure combinations. The accuracy rates in both conditions were high, but the participants were a little less accurate in detecting the related $(M=.76, S D=.16)$ than in detecting the unrelated $(M=.87, S D=.15)$ pairings $[F(1,58)=$ $25.92, p<.01]$. The analysis showed no difference in the proportion of correct responses to picture $(M=.80, S D=$ $.16)$ or word $(M=.83, S D=.17)$ primes $(F<0.1)$.

To measure whether accuracy rates in the study trials were also related to the test data, we correlated the accuracy data with recognition rates to the biased versions of the figures that were primed with related information. A relationship was not apparent, however $[r(58)=.04, p=.75]$.

\section{Discussion}

The analyses over items and over participants consistently showed successful priming effects. Presenting a prime that is somehow related to one of the alternative organizations of an ambiguous figure can influence how the participants organize and interpret the complex figures. The fact that the primed version of the figure was recognized at a higher rate than was the version of the figure that was primed with unrelated information shows the influence of the prime. The stronger recognition of the primed version relative to the unprimed version and to that primed with unrelated information was found even when response bias in the recognition memory data was controlled with the calculation of $A^{\prime}$ scores in the manner suggested by Hollingworth and Henderson (1998).

During the study phase, the participants were not told about the recognition task or about the ambiguous figures. The instructions focused the participants' attention on judging the semantic relatedness between the prime and the figure. To demonstrate the importance of this part of our procedure, we changed the orienting question in the next experiment to one that focused on a superficial aspect of the stimulus processing and tested for the priming effect with the same stimulus materials.

\section{EXPERIMENT 3}

In this experiment, we used an orienting question during the study phase that focused attention on the physical characteristics of the stimuli rather than on their semantic relationship. Diverting the participants' attention away from the semantic relationship between the stimuli was expected to weaken or remove the priming effect. As in Experiment 2, a recognition memory test was used, and successful priming was measured by comparing the recognition score with the scores of biased figures that were primed with related and unrelated information.

\section{Method}

Sixty additional students ( $75 \%$ female) volunteered from the same participant pool as in the previous experiments. With the exception of the following changes in the study phase, the materials and procedure were the same as those in Experiment 2. The word primes were of a color similar to the dominant color of their matched picture primes, and the participants were asked to compare the prime with the ambiguous figure and to indicate whether the visual material that appeared on the two screens was (1) black and white, (2) colored, or (3) mixed. They used the first three keys on the numeric keypad to indicate a response. A note placed under the monitor provided a reminder of the response associations. As in Experiment 2, there were six versions of the stimulus lists to counterbalance the presentation of the three priming conditions (primed with a related stimulus, alternative version primed, primed with an unrelated stimulus) across each of the figures and the two format conditions. Ten participants were randomly assigned to each of the lists.

\section{Results}

A between-experiments comparison of the response times to the orienting questions in the study trials showed faster responses to the question about the color of the stimuli $\left[F(1,116)=21.44, p<.01, \eta^{2}=.16\right]$. The means for Experiments 2 and 3 were 4,859 $\mathrm{msec}(S D=3,083)$ and $2,759 \mathrm{msec}(S D=1,724)$, respectively. The participants spent more time studying the ambiguous figures when the orienting question was about the relatedness between the stimuli than when superficial details were questioned. Response times were not found to differ, however, as a function of the format of the primes $[F(1,116)=3.07$, $p=.08]$, and there was no interaction of format and experiment $(F<1)$.

Table 3 reports the mean recognition scores for each of the experimental conditions averaged across participants. When these data were combined with the data from the 
Table 3

Recognition Responses for Each of the Experimental Conditions in Experiment 3

\begin{tabular}{lccccc}
\hline \multirow{2}{*}{ Condition } & \multicolumn{2}{c}{ Picture Prime } & & \multicolumn{2}{c}{ Word Prime } \\
\cline { 2 - 3 } \cline { 6 - 6 } \multicolumn{1}{c}{} & $M$ & $S D$ & & $M$ & $S D$ \\
\hline Primed & 3.77 & 0.70 & & 3.63 & 0.77 \\
Alternative primed & 3.65 & 0.74 & & 3.49 & 0.82 \\
Unrelated primed & 3.84 & 0.70 & & 3.47 & 0.82 \\
New shape & 2.18 & 0.60 & & 1.45 & 0.61 \\
New item & 2.02 & 0.63 & & 1.60 & 0.73 \\
\hline
\end{tabular}

Note-Recognition responses were measured on a 6-point response scale ( 1 , very confident no; 2 , somewhat confident no; 3 , not very confident no; 4, not very confident yes; 5 , somewhat confident yes; 6 , very confident yes).

previous experiment, the analysis showed a significant interaction of experiment and test condition $[F(4,464)=$ $\left.7.23, p<.01, \eta^{2}=.06\right]$. However, there was no main effect of experiment $[F(1,116)=1.08, p=.30]$, and experiment did not interact with format $[F(1,116)=1.07, p=.30]$ or with format and prime condition $(F<1)$. To understand how the test condition effect in Experiment 3 varied from the effect obtained in Experiment 2, follow-up analyses were concentrated on the data from Experiment 3.

In the analysis across participants, there was a significant effect of condition $\left[F_{1}(4,232)=244.28, p<.01\right.$, $\left.\eta^{2}=.81\right]$. However, the within-groups contrasts at the $p<.05$ level of significance showed that the only difference was in the comparison of the new figures (new items and new shapes) with the other conditions. Recognition of new items did not differ from that of new shapes, however, and the participants' recognition of the new figures was significantly lower than their recognition of the biased versions of the figures. In contrast to the findings of the previous experiment, there was no difference between the recognition of the biased version when primed with either a related or an unrelated stimulus and when primed with the alternative version of the ambiguous figure. Similarly, in the analysis across items, there was no effect of prime condition $\left(F_{2}<1\right)$; biased versions of the ambiguous figures were recognized with the same degree of confidence whether primed with a related $(M=3.69)$ or an unrelated $(M=3.65)$ stimulus and when compared with the recognition rate of the alternative version $(M=3.57)$ of the ambiguous figure.

The analyses of the data from Experiment 3 also differed from those of the previous experiment by showing a small main effect of format $\left[F_{1}(1,58)=6.40, p<.01\right.$, $\left.\eta^{2}=.10 ; F_{2}(1,35)=5.49, p<.05, \eta^{2}=.14\right]$. Recognition rates were higher when picture rather than word primes were used during the study trials. Format was also found to interact with test condition but only in the analysis across participants $\left[F_{1}(4,232)=3.39, p<.01, \eta^{2}=.06\right.$; $\left.F_{2}(2,70)=1.12, p=.33\right]$. The format effect was larger with recognition responses to the new shapes than in any of the other test conditions. Since the analysis across items did not include the new figures, it is not surprising that the interaction failed to reach significance.

As in the previous experiment, the participants were asked after the experimental session whether they were aware that ambiguous figures were used during the study phase. Thirteen percent indicated that they were unaware. However, when their data were analyzed separately, the pattern of findings was the same as that obtained with all of the participants. New figures were recognized at somewhat lower levels of confidence than the biased versions of the figure, but there was no evidence for a priming effect and no difference in recognition between the new items and the new shapes.

Study trial responses. Accuracy in responding to the orienting question was analyzed with a format $X$ prime condition analysis. The proportion of correct responses did not differ significantly as a function of format $\left[F_{1}(1,58)=\right.$ $3.14, p=.08]$ or condition $\left[F_{1}(2,116)=1.93, p=.15\right]$, and their interaction was not significant $(F<1)$. On average, the participants responded correctly $64 \%$ of the time $(S D=0.32)$ in the study trials. A correlation between study time accuracy and recognition responses to the biased versions that were primed with related information showed no evidence of a relationship $[r(58)=.05, p=.73]$.

\section{Discussion}

Focusing the participants' attention on the superficial aspects of the stimuli during the study phase of the experiment was sufficient to eliminate the priming effect. Even though the prime conditions were identical to those in the previous experiment, and the primes were related to one of the two interpretations of the ambiguous figures, the fact that the participants' attention was diverted away from the relatedness and focused on the color of the stimuli diminished the priming effect.

\section{GENERAL DISCUSSION}

Our findings demonstrate that both the semantic content of the prime and the attention paid to the association between the prime and the ambiguous figure are necessary for effective priming. Primes that are loosely and indirectly associated with one of the two organizations of an ambiguous figure can be effective at biasing the way a participant encodes or represents a complex figure but only if attention is focused on the semantic relationship between the two stimuli. Diverting the participants' attention to the physical characteristics of the stimuli during encoding eliminated the prime's influence on complex object perception. This result is consistent with those of other research demonstrating the effect of attention on priming effects with implicit tasks (Mulligan \& Peterson, 2008) and, more important, with those of the conceptual priming literature (Farah, 1989; Tulving \& Schacter, 1990), which suggests that semantic priming effects are distinguished from direct perceptual effects because of the involvement of memory. The fact that deep rather than superficial processing was required to show the priming effects is evidence against an early perceptual basis to the priming effect. Also, the fact that there was a higher recognition rate to the new versions of the primed shape than to the new items in Experiment 2 also shows that recognition responses were affected to 
some modest degree by the participants' memory for the primed shape. The effect was particularly apparent with picture primes.

In general, these findings support and extend Balcetis and Dale's (2007) research that showed that loosely related information modulates object identification. Even though Balcetis and Dale did not manipulate depth of processing, they did require their participants to read several paragraphs of related material, and the fact that several related paragraphs were read may be considered a form of deep processing. Also, the fact that we found successful priming with both presentation formats suggests a conceptual basis for the effect. If successful priming was caused by language factors, verbal priming should have been more effective than visual, or, alternatively, had visual priming been found to be more effective, it may also have suggested a perceptual effect that was specific to the way that pictures are represented and processed. Similar to the findings of Matsukawa et al. (2005), our results show that the picture or word format of the prime is not an important factor. The priming effect that we observed appears to result from processing the content of the prime. Format effects were only obtained in Experiment 3, in which the orienting question in the study trials focused attention on the color of the stimuli. For all of the other findings, the results were consistent across the picture and word formats.

The fact that format was not found to influence the priming effect differed from the results of some past studies with ambiguous figures (e.g., Leeper, 1935) in which more consistent effects with visual than with verbal primes were found. One of the possible reasons for the difference in the findings, we believe, is that our design controlled for a variety of linguistic and perceptual biases that were typically associated with ambiguous figure studies. We used a recognition rate as a nonlinguistic measure to assess priming effects to avoid a verbal bias in collecting the perceptual response. Also, by priming both interpretations of the ambiguous figures, counterbalancing the primed version across participants, and analyzing the data across both participants and figures, we observed a response pattern that could be generalized to a whole category of complex figures that involved figure-ground reversals and reconstrual of parts of the figure. Moreover, we minimized past experience with the figures and did not mention that the figures could be viewed in one of two ways. The participants could organize the figures in a manner that best suited their purpose without the perceptual and linguistic biases that are associated with the task.

The priming effects were obtained even though most of the participants indicated on the posttest that they were aware of the use of ambiguous figures in the study. Because other researchers (Girgus et al., 1977; Rock \& Mitchener, 1992) found that knowledge of the alternative interpretations of the figures is an important factor in determining whether the participants see the reversal, we expected that priming effects would diminish for those who experienced the reversal during the study phase of the experiment. Seeing the reversal would have provided an opportunity to encode both organizations of the complex figures, and as a result, false alarms to the biased versions would have been similar, irrespective of their relationship to the prime. However, it is somewhat problematic to assume anything more about our participants' response to the posttest question, other than an acknowledgment of some recognition of the complex nature of the stimuli used in the experiments. There is no guarantee that the participants experienced the reversal during the study trials. They may have realized the dual nature of the stimulus figures from the makeup of the test material. All that we can conclude is that the priming effects were consistent, irrespective of the participants' awareness of the use of ambiguous figures.

These findings may also extend the work of Feist and Gentner (2007) by showing that the priming effects are not specific to linguistic materials. Primes presented in both word and picture formats can interact with complex objects to influence the way in which the material is represented and remembered. Feist and Gentner's interactive encoding hypothesis may apply when either visual or verbal materials are used as primes. The interpretation of complex figures can be biased by the advanced presentation of related information; effective priming with such indirect primes only occurs when attention is focused on the semantic relationship between the prime and the complex figure, and it occurs in a similar way for both verbal and visual primes.

The interpretation of our findings is limited, however, by the fact that there was a difference between the results of Experiments 2 and 3 in the amount of time that the participants studied the ambiguous figures, because responses took longer to deep orienting questions than to shallow questions. It is reasonable to speculate about whether the presence or absence of the priming effect was a result of the study time difference between Experiments 2 and 3 or whether the findings may have been different if study time had been fixed. Although study time could have affected the overall recognition rate for the biased versions of the ambiguous figures, there is no reason to expect that it should have influenced recognition for only the version of the figure primed with a related stimulus and not for the other two biased versions. The analysis of the recognition data from both experiments (Tables 2 and 3 ) shows comparable recognition rates for the biased versions of the ambiguous figures with one significant exception: the related primed versions. As was indicated previously in our discussion of the priming effect, recognition was higher for the related primed version in Experiment 2 than for that in Experiment 3. The only other between-experiments difference was in the false alarm rates for new figures (new shapes and new items). They were higher in Experiment 3 than in Experiment 2, and this difference may possibly result from the difference in study time. The longer the time spent with the ambiguous figures, the better the participants were at discriminating elements of the complex figures from the new pictures.

It is also possible that the longer viewing time could have given the participants a chance to look for alternative interpretations of the ambiguous figures, because there were more opportunities for eye movements to mediate 
the effect. However, the fact that recognition of the biased versions that were either unprimed or primed with an unrelated prime was similar in the two experiments is evidence against this interpretation. Also, when asked whether they were aware of the ambiguous figures in the study trials, a majority of both groups of participants responded positively. Had study time been a factor in viewing the alternative interpretations of the ambiguous figures, as was suggested above, more participants would have shown a positive response in Experiment 2 rather than in Experiment 3 . A follow-up study in which limited exposure duration for the ambiguous figures is used would be needed, however, to resolve this issue.

This work makes a contribution in two notable ways: It provides some normative data about the relatedness between objects and object names and the alternate organizations of ambiguous figures, and it shows that when participants are required to process the stimulus material semantically, either objects or their names can effectively prime the interpretation of complex objects.

\section{AUTHOR NOTE}

This research was supported in part by Grant SES-0552160 from the National Science Foundation. Thanks are due Heidi Smith, Angela Dimmett, Laura Capps, Kendra Delaine, and Stacey Moran for their assistance with data collection and data analysis. Correspondence concerning this article should be addressed to P. Goolkasian, Department of Psychology, University of North Carolina, Charlotte, NC 28223 (e-mail: pagoolka@uncc.edu).

\section{REFERENCES}

Balcetis, E., \& Dale, R. (2007). Conceptual set as a top-down constraint on visual object identification. Perception, 36, 581-595. doi: $10.1068 / \mathrm{p} 5678$

Bar, M., \& Biederman, I. (1998). Subliminal visual priming. Psychological Science, 9, 464-469. doi:10.1111/1467-9280.00086

BorING, E. G. (1930). A new ambiguous figure. American Journal of Psychology, 42, 444. doi: $10.2307 / 1415447$

BotwINICK, J. (1961). Husband and father-in-law: A new reversible figure. American Journal of Psychology, 74, 312-313. doi:10.2307/1419424

Bugelski, B. R., \& Alampay, D. A. (1961). The role of frequency in developing perceptual sets. Canadian Journal of Psychology, 15, 205211. doi: $10.1037 / \mathrm{h} 0083443$

Craik, F. I. M., \& Lockhart, R. S. (1972). Levels of processing: A framework for memory research. Journal of Verbal Learning \& Verbal Behavior, 11, 671-684. doi:10.1016/S0022-5371(72)80001-X

Craik, F. I. M., \& Tulving, E. (1975). Depth of processing and the retention of words in episodic memory. Journal of Experimental Psychology: General, 104, 268-294. doi:10.1037/0096-3445.104.3.268

FARAH, M. J. (1989). Semantic and perceptual priming: How similar are the underlying mechanisms? Journal of Experimental Psychology: Human Perception \& Performance, 15, 188-194. doi:10.1037/0096 $-1523.15 .1 .188$

FEIST, M. I., \& GenTNER, D. (2007). Spatial language influences memory for spatial scenes. Memory \& Cognition, 35, 283-296.

Girgus, J. J., Rock, I., \& Egatz, R. (1977). The effect of knowledge of reversibility on the reversibility of ambiguous figures. Perception \& Psychophysics, 22, 550-556.

GoolKasian, P. (1987). Ambiguous figures: Role of context and critical features. Journal of General Psychology, 114, 217-228.

Grier, J. B. (1971). Nonparametric indexes for sensitivity and bias: Com- puting formulas. Psychological Bulletin, 75, 424-429. doi:10.1037/ h0031246

Hollingworth, A., \& Henderson, J. M. (1998). Does consistent scene context facilitate object perception? Journal of Experimental Psychology: General, 127, 398-415.

Intraub, H., \& NickLos, S. (1985). Levels of processing and picture memory: The physical superiority effect. Journal of Experimental Psychology: Learning, Memory, \& Cognition, 11, 284-298. doi:10.1037/0278-7393.11.2.284

LEEPER, R. (1935). A study of a neglected portion of the field of learning: The development of sensory organization. Journal of Genetic Psychology, 46, 41-75.

LONG, G. M., \& ToPPINO, T. C. (2004). Enduring interest in perceptual ambiguity: Alternating views of reversible figures. Psychological Bulletin, 130, 748-768. doi:10.1037/0033-2909.130.5.748

Matsukawa, J., Snodgrass, J. G., \& Doniger, G. M. (2005). Conceptual versus perceptual priming in incomplete picture identification. Journal of Psycholinguistic Research, 34, 515-540. doi:10.1007/ s10936-005-9162-5

Mulligan, N. W., \& Peterson, D. (2008). Attention and implicit memory in the category-verification and lexical decision tasks. Journal of Experimental Psychology: Learning, Memory, \& Cognition, 34, 662679. doi:10.1037/0278-7393.34.3.662

NeCKeR, L. A. (1832). Observations on an optical phenomenon which occurs on viewing a figure of a crystal or geometrical solid. Philosophical Magazine, 1, 329-337.

Peterson, M. A. (1993). The ambiguity of mental images: Insights regarding the structure of shape memory and its function in creativity. In B. Roskos-Ewoldson, M. J. Intons-Peterson, \& R. F. Anderson (Eds.), Imagery, creativity, and discovery: A cognitive perspective (pp. 151185). Amsterdam: North-Holland.

Peterson, M. A., \& Gibson, B. S. (1994). Must figure-ground organization precede shape recognition? An assumption in peril. Psychological Science, 5, 253-259. doi:10.1111/j.1467-9280.1994.tb00622.x

Peterson, M. A., Harvey, E. M., \& Weidenbacher, H. J. (1991). Shape recognition contributions to figure-ground reversal: Which route counts? Journal of Experimental Psychology: Human Perception \& Performance, 17, 1075-1089. doi:10.1037/0096-1523.17.4.1075

Ramponi, C., Richardson-Klavehn, A., \& Gardiner, J. M. (2007). Component processes of conceptual priming and associative cued recall: The roles of preexisting representation and depth of processing. Journal of Experimental Psychology: Learning, Memory, \& Cognition, 33, 843-862. doi: $10.1037 / 0278-7393.33 .5 .843$

Rock, I., \& Mitchener, K. (1992). Further evidence of failure of reversal of ambiguous figures by uninformed subjects. Perception, 21, 39-45. doi:10.1068/p210039

Rubin, E. (1958). Figure and ground. In D. C. Beardslee \& M. Wertheimer (Eds. \& Trans.), Readings in perception (pp. 35-101). Princeton, NJ: Van Nostrand.

Tulving, E., \& Schacter, D. L. (1990). Priming and human memory systems. Science, 247, 301-306. doi:10.1126/science.2296719

Zwaan, R. A., Stanfield, R. A., \& Yaxley, R. H. (2002). Language comprehenders mentally represent the shapes of objects. Psychological Science, 13, 168-171. doi:10.1111/1467-9280.00430

\section{NOTE}

1. A conventional analysis of the recognition data was also conducted by converting the rating scale data into proportions of yes and no responses, with ratings of 3 or less scored as no and of greater than 3 as yes. The $2 \times 5$ ANOVA on the proportion of yes responses showed the same pattern of findings as the analysis on the rating scale data; that is, figures primed with a related stimulus were recognized at a higher rate than figures primed with an unrelated prime or when the alternative version was primed. There was a significant effect of test condition $[F(4,232)=$ $237.25, p<.01]$, but no effect of format $[F(1,58)=2.38, p=.13]$ and no interaction of format and condition $[F(4,232)=1.26, p=.29]$. 
List of Stimulus Materials

\begin{tabular}{|c|c|c|}
\hline Ambiguous Figure & Prime Version 1 & Prime Version 2 \\
\hline Woman/old woman & Woman's long curly hair & $\begin{array}{l}\text { Old woman with long nose, woman } \\
\text { with cane }\end{array}$ \\
\hline Woman/skeleton & $\begin{array}{l}\text { Young lady looking in a mirror, hand } \\
\text { mirror }\end{array}$ & Skeleton, tombstone \\
\hline Swan/squirrel & Bird soaring, duck in water & Acorn, squirrel feeder \\
\hline Sax/woman & Trumpet, music notes & Young lady in a dress, woman speaker \\
\hline Rabbit/duck & Rabbit ears, bunch of carrots & Mallard duck, goose \\
\hline Profile/cup & Profile of face, facial features & Drinking glass \\
\hline Plane/bird & $\begin{array}{l}\text { Paper airplane, airplane landing, con- } \\
\text { trol panel of airplane }\end{array}$ & Small red and yellow bird \\
\hline Liar/face & Words on a page & Eyeglasses, businessmen \\
\hline Moon/sign & Sun, astronaut on moon & Road signs, driving signs \\
\hline Lift/puzzle & Words on a page & Puzzle pieces \\
\hline Lady/man & Lady seated on floor & Businessman, man's face \\
\hline Husband/father & Handsome man, young men & Older men with hats \\
\hline Horse/seal & Zebra, barn & Penguin, water animals \\
\hline Horse/old man & Cowboy on a horse, cowboy hat & Bearded old men \\
\hline Face/butterfly and flower & Lips, eyes & Butterfly, pink rose, flower \\
\hline Eskimo/Indian & Young boy, winter scene & Peacock feathers, tomahawk \\
\hline Duck/rabbit & Mallard duck, goose & Rabbit ears, bunch of carrots \\
\hline Left arrow/right arrow & Left arrow, yellow & Right arrow \\
\hline
\end{tabular}

(Manuscript received May 11, 2009;

revision accepted for publication July 20, 2009.) 